\title{
An Automatic Apparel Designing System Based on Databases
}

\author{
Yue Li, Xiaogang Wang* and Xinzhan Li \\ Wuhan Textile University, Wuhan City, Hubei Province, China, 430073 \\ ${ }^{*}$ Corresponding author
}

\begin{abstract}
In this article based on databases of 3D female bodies emulator, 3D jacket emulator, textile material parameters, historical photos and pattern parameters, an automatic pattern making and its 3D emulator model support system of woman warm jacket was developed. In this system different databases were set up and mathematic models of body block and pattern were founded, which they were the main algorithms of program. Program of connecting databases for pattern making was developed and its 3D emulator model was realized synchronization. Based on 20 variables for the female body, the net body block is first be made for individuality and its $3 \mathrm{D}$ model also is offered. Then according to the parameters from textile material database and pattern database, pattern is automatically made on the base of the body block. At the same time its 3D jacket model can be presented to user by this system. In this system, managing and connecting different types of databases were come true. This new automatic designing method differs with the traditional pattern making, which introduced the database technology to make works automatically and present 3D emulator model synchronously. This open system is offered user with spreading the database possible and connecting with ready CAD system.
\end{abstract}

Keywords-apparel design; database; 3D emluator model; automatic design

\section{INTRODUCTION}

As usual, there are problems that many design idea are incomplete, inaccurate and inconsistent to be transferred between designers and technicians[1]. The communication between designers and technicians poses a serious bottleneck even if they work closely. Though apparel CAD systems available have been very perfect, it is longer time for designer or pattern maker to make pattern step by step. Thus the mode of garment manufacture is facing an immensity change under the driving of computer technology. With the development of CAD technology, the expert system gradually has been making for apparel manufacture bottleneck---pattern making. Besides the expert system is applied to make pattern automatically, which can create new pattern automatically according to the information of consumer.[2] Some crafts that depend on specialized worker can be stabilized to improve the efficiency of production. So it is necessary to develop an automatic system for pattern making to overcome these problems. This automatic system not only helps designer finish pattern but also present the model for them. This system is the key for intelligent research in apparel science, which it must express information of human body, textile material parameters, apparel fashionable shape and pattern.

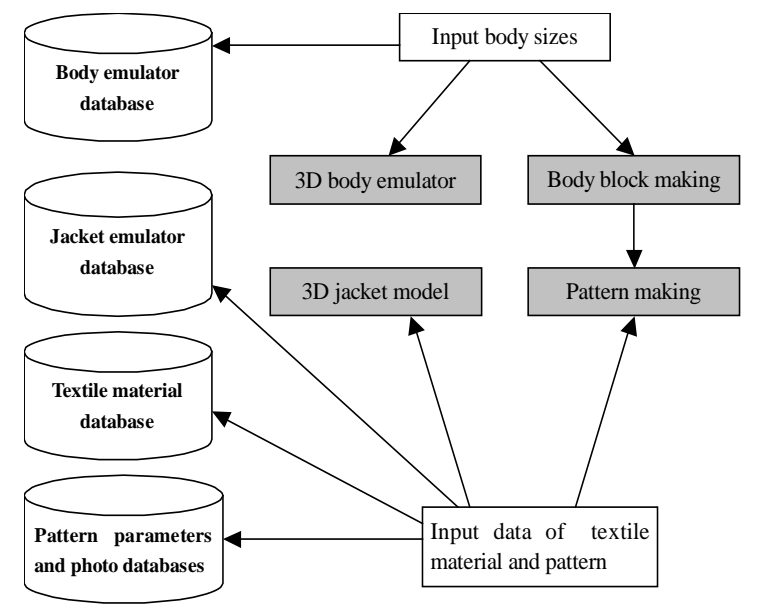

FIGURE I.

ARCHITECTURE OF AUTOMATIC PATTERN

This paper discusses how to develop an automatic pattern making system for the woman warm jacket. In this system there are different databases. Based on these databases different factors were involved for pattern, which is related with body sizes, textile material parameters, pattern indexes and its shapes. And at the same time 3D emulator model database could offer 3D model synchronously. At first body sizes are input by user, which there are 20 variables for the female body. In this step body block is made for individuality according to values of these variables, which it is the basement for the next step. The 3D body emulator model file will be output from the body emulator database simultaneity. The second step is choosing or inputting values of textile material parameters, which there are popular examples for textile materials and their parameters. Then the pattern parameters and photos database offers historical photos for each year, which they were collected from Chinese and Russian fashion journals and magazines. In this database data of 17 pattern parameters for each photo are offered for referring or deciding. As pattern is made by these information, the jacket emulator model is output from the jacket emulator database. Mathematic models of block and pattern were calculated to be the main algorithm of program. All databases were connected by program to be an automatic pattern making system, which they are open and interassembler. This expert system realized the automatic pattern making for 
woman warm jacket and presenting its 3D model. There have been our efforts to for apparel intelligent research.

\section{ARCHITECTURE OF THE SYSTEM}

Automatic pattern making system is a complex system. The method of pattern making for developing the automatic system is very important. For it is not only related with the intelligent of system, but also the flexibility for individuality. The whole architecture of the system is as Figure 1 shows. There are 4 databases including body emulator, jacket emulator, textile material, pattern parameters and photos. When body sizes are input to the system, the body block first will be made and its 3D model also will show to user. Then parameters of textile material and pattern must be input. At last pattern will be finished on the base of block and its 3D model will also present.

The first database in this system is body emulator database. Its Aim is making body block for individual body and showing its emulator model. The net body block is the block pattern for body, which there is no ease. It is necessary for 20 body sizes to make body block. Data for these parameters and their 3D models can be achieved by body scanner or measuring. For there is transferring program developed to input the scanning file in this system.

The second database in this system is textile material parameters database. As we know, when there is the interlining for woman warm jacket for keeping warm, the pattern will be enlarged for its thickness. The second step for the system developed to allow user to choose the thickness of interlining. There are several typical examples of interlining thickness, as user can also input the parameter of his production. In this database, system also put apart other parameters for fabric and interlining, which is for extending in the future. These parameters are flexibility, bending and so on.

The third database in this system is photo and pattern parameters database. There are typical fashion shapes in every year for woman warm jacket. 98 photos were collected in this database, which they are historical photos from 1978 to 2006. And fashionable information was extracted. These photos are offered to user, which their shapes are the reference. Corresponding to each photo database offers data of 17 indexes for its pattern.

The jacket emulator database offers 3D model for each pattern. The 3D jacket emulator model files can be achieved by the 3D scanner system. For these model files can be transferred and reconstructed in this system.

Of course all databases in this system are open for the user, which they permit adding, changing or deleting data. All data or value chosen by user for each database will be input into the algorithm for pattern making, which the algorithm was developed into program. The programming language was the visual $\mathrm{C}++$.

\section{MATHEMATIC MODELING OF BODY BLOCK AND PATTERN}

\section{A. Mathematic Modeling of Body Block}

As you know, the two-dimensional patterns in available apparel CAD systems were developed into vector graphs. That means there is initial coordinate to the working area in CAD system. Some points decide all lines and curves. Thus mathematic modeling of block and pattern are the work to calculate equations of characteristic point coordinates along the pattern outline. Figure 2 shows the characteristic points in front and back block.

Body block is first to be drawn by system automatically. There are initial points for front piece and back piece. They are the center points in front and back waist. Variables $\mathrm{X}^{1} \ldots \mathrm{X}^{20}$ for calculating block are as in Figure 6. Coordinates of characteristic points are calculated to be the complex function, which finish the mathematic model for the body block pattern in the first step. These models for the coordinate of each point in front and back are the main algorithms in program.
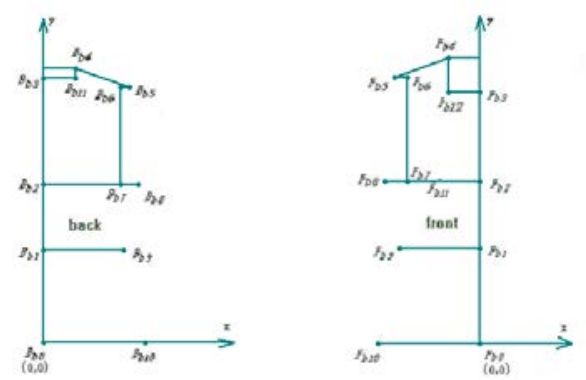

FIGURE II.

CHARACTERISTIC POINTS IN BODY BLOCK

\section{B. Addition of Interlining Thickness to Pattern}

The warm jacket is the most popular clothing in winter and is favored by customers. To make a precise pattern for fabric designers must consider thickness of its interlining. But it is not directly to add the thickness to pattern. In our research the problem was solved and also the result was developed to the program for pattern making. This automatic calculating by program improves the pattern precision.

As usual patterns are made into front and back. For female body, there are two angles used in the calculation of thickness, which they are the back slope angle and bust slope angle as in Figure 6. Figure 3 show that these two angles are different. We can see that pattern for the fabric differ with that for the lining, which there is interlining thickness. And the addition to pattern from interlining lies on these angles and its thickness. The following equation 1 and 2 list the process for the addition to the length in front and back.
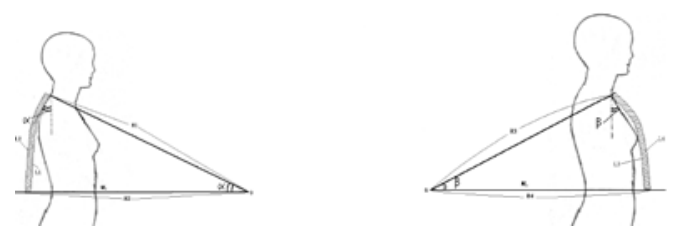

FIGURE III. THICKNESS OF INTERLINING ADDED TO THE LENGTH IN BACK AND FRONT PATTERN 
For back:

$$
L_{2}-L_{1}=\frac{\alpha \pi}{180}\left(R_{2}-R_{1}\right)=\frac{\alpha \pi H}{180}
$$

Where: $R_{2} R_{1}=\mathrm{H}, \mathrm{H}$ stands the thickness of interlining, $\mathrm{L}^{2}$-L ${ }^{1}$ stands the addition of interlining thickness to back pattern length.

For front:

$$
L_{4}-L_{3}=\frac{\alpha \pi}{180}\left(R_{4}-R_{3}\right)=\frac{\beta \pi H}{180}
$$

Where: $R_{4} R_{3}=\mathrm{H}, \mathrm{H}$ stands the thickness of interlining, $\mathrm{L}^{4}-\mathrm{L}^{3}$ stands the addition of interlining thickness to front pattern length.

The thickness of interlining influences not only on the length of fabric pattern, but also it influences on the girth of fabric pattern. As Figure 4 shows that the female body is regarded as an ellipse, the addition for the girth can be calculated as the following equation 3[3]. These results will be connected with the latest pattern mathematic model.

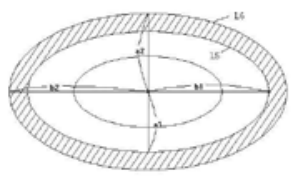

FIGURE IV.

THICKNESS OF INTERLINING ADDED TO PATTERN

$$
\mathrm{L}^{6}-\mathrm{L}^{5}=\pi\left(\mathrm{a}^{2}-\mathrm{a}^{1}\right)+\pi\left(\mathrm{b}^{2}-\mathrm{b}^{1}\right)=2 \pi \mathrm{H}
$$

Where: $a^{2}-a^{1}=H, b^{2}-b^{1}=H, H$ stands the thickness of interlining, $\mathrm{L}^{6}-\mathrm{L}^{5}$ stands the addition of interlining thickness to pattern girth.

\section{Pattern Modeling}

Mathematic model for pattern outline was set up on the basis of mathematic model of body block and result of interlining thickness[4]. In another word mathematic model of pattern is the complex function as the following equation 4 shows. Thus coordinates of points in pattern are the function of the interlining thickness, body parameters and pattern parameters as the following equation 4. Where Ppi (xi, yi) stands the coordinate of point in pattern, $\mathrm{Xi}$ stands the variables of body, $\mathrm{H}$ stands the variable of interlining thickness, and vi stands variables of pattern.

$$
\text { Ppi (xi, yi) = f (Xi, H, vi) }
$$

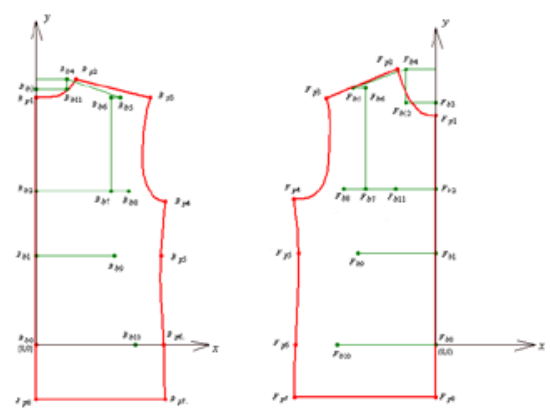

FIGURE V.

CHARACTERISTIC POINTS IN PATTERN

Figure 5 shows characteristic points in front and back patterns, which their coordinates depends on points of body block and eases in different directions. These mathematic models were the main algorithms for developing program of automatic pattern making.

\section{REALIZATION OF THE SYSTEM}

As forenamed there are several databases in the system. The program manages files in different databases. And the system calculates the outlines of body block and pattern to show. The principle of 3D shape presentation in system is that system chooses the 3D files from 3D body emulator database or jacket database according to parameters provided by user. Then the system realizes the presentation of 3D emulator model synchronously as pattern is made. The basic algorithm in program is as following.

The time complexity of computing is constant factorial.

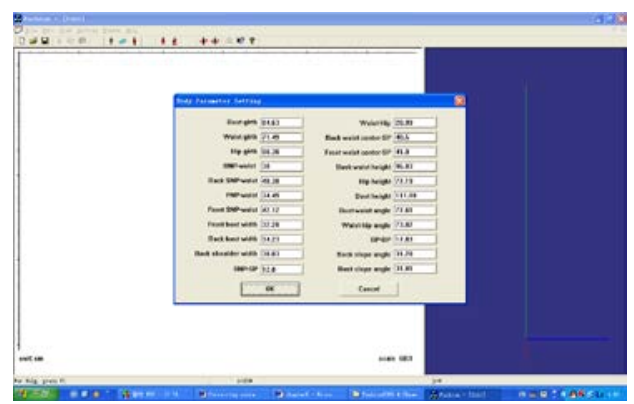

FIGURE VI.

VARIABLES WINDOW FOR BODY BLOCK IN SYSTEM

As Figure 6 shows the window in system for body parameter. Values for the default setting are statistic average of 500 female body sizes. And for each parameter there is the interval for its value. The system automatically will suggest user, when there is mistake value that is not in the usual interval. Figure 7 is the result of running program, which body block is made and its 3D emulator model is presented. It is made from body sizes and will be the basement for pattern. 


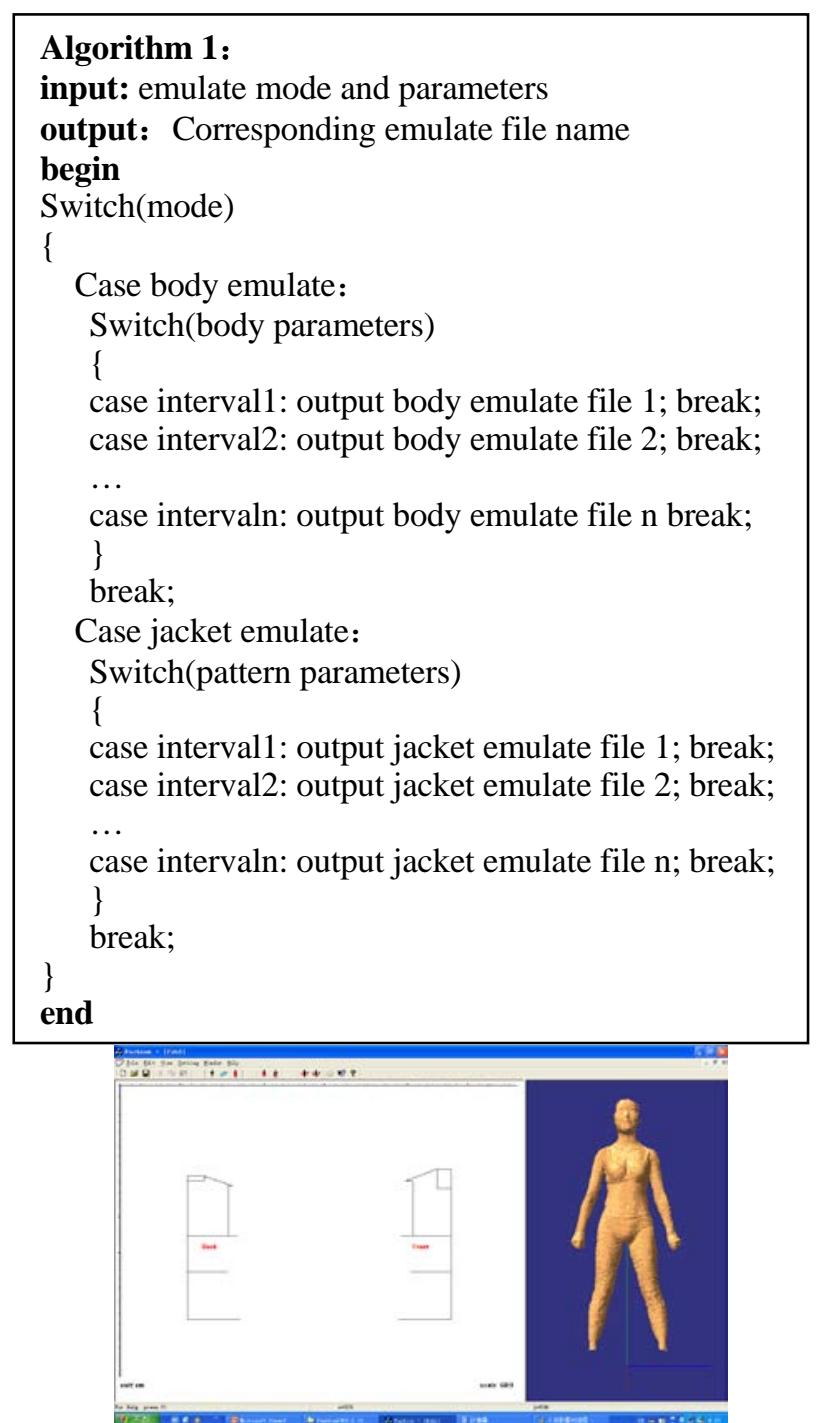

FIGURE VII

BODY BLOCK AND ITS 3D MODEL MADE BY THE AUTOMATIC SYSTEM

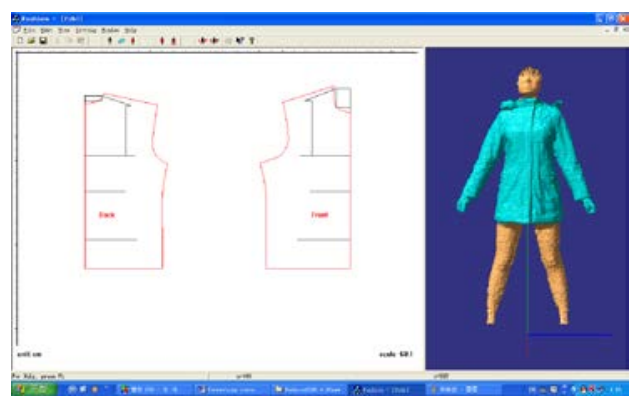

FIGURE VIII.

PATTERN AND 3D MODEL MADE BY THE AUTOMATIC SYSTEM

When system gains parameters of textile material and pattern, the last pattern is drawn by calculating the geometry characteristic points according to the mathematic models that mentioned above. Pattern is made and its 3D emulator model presents in Figure 8.

\section{CONCLUSIONS}

Based on databases of body emulator, textile material parameters, jacket emulator, pattern parameters and historical photos, an automatic system for pattern making and presenting its 3D emulator model was developed for woman warm jacket. Different databases were set up and mathematic models of body block and pattern were calculated to be the main algorithm for program. By intelligent completion of databases, automatic solution of pattern and presenting 3D emulator model are generated by this system.

This automatic pattern making system improves research about the expert system and also extends the apparel CAD, which can be connected with ready CAD system. The whole thinking, different databases setting up and connecting also broke a new channel to intelligent technology in apparel science[5]. The automatic pattern making is also for other sort of apparel. The result that we achieved has the potential to become a key component in expert system.

\section{ACKNOWLEDGEMENT}

Research was supported in part by the Science Foundation of Hubei Province China grant number, 2014CFB761, 2015CFB429 and the Science and Technology Guiding Project of Education Department of Hubei China grant number D20121704, B2015312.

\section{REFERENCES}

[1] M Li, L Ma, A Study on Bodice Prototype \& Its Pattern Design System, Journal of Donghua University Natural Science, Vol.12 (2),( 2002)34-36.

[2] Ji-Young Lim, A Study on the Ease in Jacket Bodice Pattern for Abdomen-obese Middle-aged Men from Virtual Appearance Evaluation, Journal of the Korean Society for Clothing Industry, Vol .12 (6) (2010)54-60.

[3] Claudia M.Eckert, Helmut E.Bez, Loughborough, UK, A garment design system using constrained Bézier curves, International journal of clothing science and technology, Vol.12 (2),(2000)34-36.

[4] Feng Ji, Ruqin Li, and Yiping Qiu, Three-dimensional Garment Simulation Based on a Mass-Spring System, Textile Research Journal, Vol76 (1), (2006)12-17.

[5] Dai wei, Zhang weiyuan, Fuzz mathematic methodology clothing fitness evaluation, Journey of Donghua university, Vol.29(3), (2003)156-159. 